\title{
On Generalized Order of Vector Dirichlet Series of Fast Growth
}

\author{
Wanchun Lu \\ Department of Mathematics, Pingxiang University, Pingxiang, China \\ Luwanchun540@163.com
}

\begin{abstract}
The concept of vector valued Dirichlet series was introduced by B. L. Srivastava [2] who characterized the growth of entire functions represented by these series. In this paper we introduce the generalized order of analysis functions fast growth.
\end{abstract}

Keywords-Vector valued dirichlet series; Analysis functions; Generalized order; Fast growth.

\section{INTRODUCTION}

Let

$$
f(s)=\sum_{n=0}^{+\infty} a_{n} e^{-\lambda_{n} s},(s=\sigma+i t, \sigma, t \in \mathbb{R})
$$

Where $a_{n}$ 's belong to a complex commutative Banach algebra $B$ with identity element $\|\omega\|=1$ and $\lambda_{n}{ }^{\prime} s \in \mathbb{R}$ satisfy the conditions $0=\lambda_{0}<\lambda_{1}<\lambda_{2}<\cdots<\lambda_{n} \uparrow+\infty$

$$
\varlimsup_{n \rightarrow+\infty} \frac{\log \left\|a_{n}\right\|}{\lambda_{n}}=0, \varlimsup_{n \rightarrow+\infty} \frac{n}{\lambda_{n}}=D<+\infty,
$$

Then, the vector valued Dirichlet series in (1) represents an analytic function $f(s)$ in right plane (see [1]). For the vector valued analytic function $f(s)$ defined as above by (1) the maximum modulus, the maximum term and the index of maximum term are defined as

$$
\begin{gathered}
M(\sigma, f)=\sup _{-\infty<t<+\infty}\{\|f(\sigma+i t)\|\} \\
m(\sigma, f)=\max _{n \in N}\left\{\left\|a_{n}\right\| e^{-\lambda_{n} \sigma}\right\} .
\end{gathered}
$$

The order $\rho$ of $f(s)$ is defined as

$$
\rho=\varlimsup_{\sigma \rightarrow 0} \frac{\log ^{+} \log ^{+} M(\sigma, f)}{-\log \sigma} .
$$

We shall call the vector valued analytic function $f(s)$ to be of fast growth if the order $\rho=\infty$. We obtain the characterization of growth parameters in the context of generalized order of vector valued Dirichlet series of fast growth.
Let $\Delta_{0}$ be the class of all functions $\beta$ satisfying the following two conditions:

(i) $\beta(x)$ is defined on $[a, \infty), a>0$, and is positive, strictly increasing, differentiable and tends to $\infty$ as $x \rightarrow \infty$;

(ii) $\frac{\mathrm{d} \beta(x)}{\mathrm{d} \log x}=o(1)$ as $x \rightarrow \infty$.

For a vector valued analytic function $f(s)$ given by (1) and $\beta(x) \in \Delta_{0}$, set

$$
\rho(\beta, f)=\varlimsup_{\sigma \rightarrow 0} \frac{\beta\left(\log ^{+} M(\sigma, f)\right)}{-\log \sigma}
$$

Then $\rho(\beta, f)$ will be called, respectively, $\beta$-order of $f(s)$. To avoid some trivial cases we shall assume throughout that $M(\sigma, f) \rightarrow \infty$ as $\sigma \rightarrow 0$.

\section{MAIN RESULTS}

Lemma 2.1 If the vector valued Dirichlet series given by (1) satisfies (2), then

$$
m(\sigma, f) \leq M(\sigma, f) \leq K(\varepsilon) m(\sigma(1-\varepsilon), f) \frac{1}{\sigma},
$$

Where $K(\varepsilon)$ is a positive number of $\varepsilon$ and $f(s)$.

Proof: From the second equation of (2), for given $\varepsilon>0$, there exists an $n_{0}=n_{0}(\varepsilon)$, such that for $n>n_{0}$, $n<(D+\varepsilon) \lambda_{n}$. Taken $\sigma<0, \varepsilon \in(0,-\sigma)$, we have

$$
\begin{gathered}
M(\sigma, f) \leq \sum_{n=1}^{n_{0}}\left\|a_{n}\right\| e^{-\lambda_{n} \sigma}+\sum_{n=n_{0}}^{\infty}\left\|a_{n}\right\| e^{-\lambda_{n}(1-\varepsilon) \sigma} e^{-\lambda_{n} \varepsilon \sigma} \\
\leq n_{0} m(\sigma, f)+m((1-\varepsilon) \sigma, f) \sum_{n=n_{0}+1}^{\infty} e^{-n \varepsilon \sigma /(D+\varepsilon)} \\
<n_{0} m(\sigma, f)+\frac{m((1-\varepsilon) \sigma, f)}{1-e^{-\varepsilon \sigma /(D+\varepsilon)}}
\end{gathered}
$$

The lemma now follows from above and the well known relation $m(\sigma, f) \leq M(\sigma, f)$

Theorem 2.1 If the vector valued Dirichlet series given by (1) satisfies (2), then

$$
\rho(\beta, f)=\varlimsup_{\sigma \rightarrow 0} \frac{\beta\left(\log ^{+} m(\sigma, f)\right)}{-\log \sigma}
$$


Proof: By the first inequality of Lemma 2.1, this gives, since $\beta \in \Delta_{0}$, that

$$
\varlimsup_{\sigma \rightarrow 0} \frac{\beta\left(\log ^{+} m(\sigma, f)\right)}{-\log \sigma} \leq \varlimsup_{\sigma \rightarrow 0} \frac{\beta\left(\log ^{+} M(\sigma, f)\right)}{-\log \sigma}
$$

By the second inequality of Lemma 2.1, we have

$$
\begin{gathered}
\log ^{+} M_{u}(\sigma, F) \\
\leq \log ^{+} m(\sigma(1-\varepsilon), f)-\log \sigma+O(1) \\
\leq C \log ^{+} m(\sigma(1-\varepsilon), f) \cdot(-\log \sigma)
\end{gathered}
$$

For all $\sigma(\sigma>0)$ sufficiently close to 0 . Here $C$ is a constant. Now, (4) gives

$$
\beta\left(\log ^{+} M(\sigma, f)\right)
$$

$\leq \beta\left(\log ^{+} m(\sigma(1-\varepsilon), m)\right)+\left.\log \left((-\log \sigma)^{C}\right) \cdot \frac{\mathrm{d} \beta(x)}{\mathrm{d} \log x}\right|_{x=x^{*}(\sigma)}$

Where $\log ^{+} \mu(\sigma(1-\varepsilon), F)<x^{*}(\sigma)<C \log ^{+} \mu(\sigma(1-\varepsilon), F)$. $(-\log \sigma)$. This easily gives

$$
\varlimsup_{\sigma \rightarrow 0} \frac{\beta\left(\log ^{+} M(\sigma, f)\right)}{-\log \sigma} \leq \varlimsup_{\sigma \rightarrow 0} \frac{\beta\left(\log ^{+} m(\sigma, f)\right)}{-\log \sigma}
$$

The theorem follows from (3) and (5).

Theorem 2.2 If the vector valued Dirichlet series given by (1) satisfies (2) and has $\beta$-order $\rho(\beta, f)$, then

$$
\rho(\beta, f)=\varlimsup_{n \rightarrow \infty} \frac{\beta\left(\lambda_{n}\right)}{\log \lambda_{n}-\log ^{+} \log \left\|a_{n}\right\|}
$$

Proof: Let $\varlimsup_{n \rightarrow \infty} \frac{\beta\left(\lambda_{n}\right)}{\log \lambda_{n}-\log ^{+} \log \left\|a_{n}\right\|}=\theta$. Clearly $0 \leq \theta \leq \infty$. First let $0<\theta<\infty$. Then, for $0<\varepsilon<\theta$ there exist a sequence $\left\{n_{k}\right\} \subset \mathbb{N}$ such that

$$
\log \left\|a_{n_{k}}\right\|>\lambda_{n_{k}} \exp \left\{-\frac{\beta\left(\lambda_{n_{k}}\right)}{\theta-\varepsilon}\right\}, k=1,2,3, \cdots \text {. }
$$

By Lemma 2.1, we have $\log ^{+} M(\sigma, f) \geq \log ^{+} m(\sigma, f) \geq \log \left\|a_{n_{k}}\right\|-\sigma \lambda_{n_{k}}$

$$
>\lambda_{n_{k}} \exp \left\{-\frac{\beta\left(\lambda_{n_{k}}\right)}{\theta-\varepsilon}\right\}-\sigma \lambda_{n_{k}}
$$

For $k=1,2,3, \cdots$, set $\sigma_{k}=\frac{1}{2} \exp \left\{-\frac{\beta\left(\lambda_{n_{k}}\right)}{\theta-\varepsilon}\right\}$. Putting, in particular, $\sigma=\sigma_{k}$ in (6), we get

$$
\log ^{+} M\left(\sigma_{k}, f\right)>\frac{1}{2} \lambda_{n_{k}} \exp \left\{-\frac{\beta\left(\lambda_{n_{k}}\right)}{\theta-\varepsilon}\right\}=\lambda_{n_{k}} \sigma_{n_{k}},
$$

Or

$\beta\left(\frac{1}{\sigma_{k}} \log ^{+} M\left(\sigma_{k}, f\right)\right)>\beta\left(\lambda_{n_{k}}\right)=(\theta-\varepsilon) \log \left(\frac{1}{2 \sigma_{k}}\right)$.

Since $\beta \in \Delta_{0}$, we have

$\beta\left(\frac{1}{\sigma_{k}} \log M\left(\sigma_{k}, f\right)\right)$

$=o(1)\left(\log \left(\frac{1}{\sigma_{k}} \log M\left(\sigma_{k}, f\right)\right)\right.$

$=o(1) \log \left(\frac{1}{\sigma_{k}}\right)+o(1) \log \log M\left(\sigma_{k}, f\right)$

$=\left.\log \left(\frac{1}{\sigma_{k}}\right) \frac{\mathrm{d} \beta(x)}{\mathrm{d} \log x}\right|_{x=x^{*}\left(\sigma_{k}\right)}+\beta\left(\log M\left(\sigma_{k}, f\right)\right)$

where $\log M\left(\sigma_{k}, f\right)<x^{*}\left(\sigma_{k}\right)<\frac{1}{\sigma_{k}} \log M\left(\sigma_{k}, f\right)$.

By (7), we have

$$
\begin{gathered}
\beta\left(\log ^{+} M\left(\sigma_{k}, f\right)\right)+\log \left(\frac{1}{\sigma_{k}}\right) \cdot \frac{\mathrm{d} \beta(x)}{\mathrm{d} \log x} \mid x=x^{*}\left(\sigma_{k}\right) \\
>(\theta-\varepsilon) \log \left(\frac{1}{2 \sigma_{k}}\right)
\end{gathered}
$$

Since $\beta \in \Delta_{0}$, dividing by $\log \left(\frac{1}{\sigma_{k}}\right)$ and passing to limits, we get

$$
\rho(\beta, f) \geq \theta
$$

(8) is obvious for $\theta=0$. For $\theta=\infty$, the above arguments with an arbitrarily large number in place of $\theta-\varepsilon$ give $\rho(\beta, F)=\infty$.

To prove the reveres inequality, since there is nothing to prove if $\theta=\infty$, we may assume that $\theta<\infty$. Then, given $\varepsilon>0$ and for all $n>n_{1}=n_{1}(\varepsilon)$ we have 


$$
\log \left\|a_{n}\right\|<\lambda_{n} \exp \left(-\frac{\beta\left(\lambda_{n}\right)}{\theta+\varepsilon}\right)
$$

Now the second equation of (2) holds, we have $n<\bar{D} \lambda_{n}$ for all $n>n_{2}=n_{2}(\bar{D})$, where $\bar{D}>D$ is a fixed constant. Let $n_{3}=\max \left\{n_{1}, n_{2}\right\}$, then from (9), we have

$$
\begin{aligned}
& M(\sigma, f) \leq \sum_{n=1}^{n_{3}} \exp \left\{\lambda_{n} \exp \left(-\frac{\beta\left(\lambda_{n}\right)}{\theta+\varepsilon}\right)-\lambda_{n} \sigma\right\} \\
& +\sum_{n=n_{3}+1}^{\infty} \exp \left\{\lambda_{n} \exp \left(-\frac{\beta\left(\lambda_{n}\right)}{\theta+\varepsilon}\right)-\lambda_{n} \sigma\right\} .
\end{aligned}
$$

For every $\sigma(\sigma>0)$ we define $n(\sigma)$ as

$$
\lambda_{n(\sigma)} \leq \beta^{-1}\left(-(\theta+\varepsilon) \log \left(\frac{\sigma}{2}\right)\right)<\lambda_{n(\sigma)+1}
$$

For $\sigma(\sigma>0)$ is sufficiently close to 0 , we have $n(\sigma)>n_{3}$. Thus, we have

$$
\begin{gathered}
\sum_{n=n(\sigma)+1}^{\infty} \exp \left\{\lambda_{n} \exp \left(-\frac{\beta\left(\lambda_{n}\right)}{\theta+\varepsilon}\right)-\sigma \lambda_{n}\right\} \\
<\sum_{n=n(\sigma)+1}^{\infty} \exp \left\{\frac{-\sigma \lambda_{n}}{2}\right\}<\sum_{n=n(\sigma)+1}^{\infty} \exp \left\{\frac{-\sigma n}{2 \bar{D}}\right\} \\
<\frac{\exp \left\{\frac{-\sigma(n(\sigma)+1)}{2 \bar{D}}\right\}}{1-\exp \left\{\frac{-\sigma}{2 \bar{D}}\right\}}=H(n(\sigma)) .
\end{gathered}
$$

Now,

$$
\begin{aligned}
& \log H(n(\sigma))=\frac{-\sigma(n(\sigma)+1)}{2 \bar{D}}+\log \frac{2}{\sigma}+O(1) \\
& <\frac{-1}{x(\sigma)} \beta^{-1}((\theta+\varepsilon) \log x(\sigma))+\log x(\sigma)+o(1), \\
& \text { Where } x(\sigma)=\frac{2}{\sigma} \text {. Clearly } x(\sigma) \rightarrow \infty \text { as } \sigma \rightarrow 0 .
\end{aligned}
$$$$
\text { Since } \beta \in \Delta_{0} \text {, it follows that } \beta^{-1}(\bar{\theta} \log x(\sigma))>(x(\sigma))^{2}
$$$$
\text { for all } \sigma \text { sufficiently close to } 0 \text {. This shows that }
$$$$
\log H(n(\sigma)) \rightarrow \infty \text { as } \sigma \rightarrow 0
$$

or

$$
H(n(\sigma)) \rightarrow 0 \text { as } \sigma \rightarrow 0 \text {. }
$$

Consider the function $\varphi(x)=x \exp \left\{-\frac{\beta(x)}{\theta+\varepsilon}\right\}-x \sigma$. Taking derivative of $\varphi(x)$ and setting it equal to 0 we get

$$
\varphi^{\prime}(x)=\exp \left\{-\frac{\beta(x)}{\theta+\varepsilon}\right\}\left(1-\frac{1}{\theta+\varepsilon} \frac{\mathrm{d} \beta(x)}{\mathrm{d} \log x}\right)-\sigma=0
$$

Since $\frac{\mathrm{d} \beta(x)}{\mathrm{d} \log x}=o(1)$ as $x \rightarrow \infty$, it follows that

$$
\frac{1}{2}<1-\frac{1}{\theta+\varepsilon} \frac{\mathrm{d} \beta(x)}{\mathrm{d} \log x}<2 \text { for } x>x_{0} .
$$

Let $\lambda_{n_{0}}$ be a fixed $\lambda_{n}$ greater than $x_{0}$ and $\lambda_{n_{3}}$, then $\varphi^{\prime}\left(\lambda_{n_{0}}\right)>0$ for $0<\sigma<\sigma_{1}$. Also $\varphi^{\prime}\left(\lambda_{n(\sigma)+1}\right)<0$ for all $0<\sigma<\sigma_{2}$. Now for $0<\sigma<\sigma_{0}=\min \left(\sigma_{1}, \sigma_{2}\right)$, we denote by $x_{*}(\sigma)$ the point where $\varphi\left(x_{*}(\sigma)\right)=\max _{\lambda_{n_{0}} \leq x \leq \lambda_{n(\sigma)+1}} \varphi(x)$, then $\lambda_{n_{0}}<X_{*}(\sigma)<\lambda_{n(\sigma)+1}$ and

$$
\begin{gathered}
x_{*}(\sigma)=\beta^{-1}\left(-(\theta+\varepsilon) \log \frac{\sigma}{1-\mathrm{d}(\sigma)}\right), \\
\mathrm{d}(\sigma)=\left.\frac{1}{\theta+\varepsilon} \frac{\mathrm{d} \beta(x)}{\mathrm{d} \log x}\right|_{x=x_{*}(\sigma)} \text {, and so } \\
\max _{n_{0} \leq n \leq n(\sigma)+1}\left\{\left\|a_{n}\right\| \mathrm{e}^{-\lambda_{n} \sigma}\right\} \leq \exp \left\{\varphi\left(x_{*}(\sigma)\right)\right\} \\
\leq \exp \left\{\beta^{-1}\left(-(\theta+\varepsilon) \log \frac{\sigma}{1-\mathrm{d}(\sigma)}\right) \frac{\sigma}{1-\mathrm{d}(\sigma)}\right. \\
\left.-\sigma \beta^{-1}\left(-(\theta+\varepsilon) \log \frac{\sigma}{1-\mathrm{d}(\sigma)}\right)\right\} \\
\leq \exp \left\{\frac{\sigma \mathrm{d}(\sigma)}{1-\mathrm{d}(\sigma)} \beta^{-1}\left(-(\theta+\varepsilon) \log \frac{\sigma}{1-\mathrm{d}(\sigma)}\right) \frac{\sigma}{1-\mathrm{d}(\sigma)}\right. \\
\leq \exp \left\{\sigma \beta^{-1}\left(-(\theta+\varepsilon) \log \frac{\sigma}{2}\right)\right\} \\
\text { Now, for } 0<\sigma<\sigma_{0}, \text { we have } \\
M_{u}(\sigma, F) \leq P\left(n_{0}\right)+\sum_{n=n_{0}}^{n(\sigma)} \exp \left\{\lambda_{n} \exp \left(-\frac{\beta\left(\lambda_{n}\right)}{\theta+\varepsilon}\right)-\lambda_{n} \sigma\right\} \\
+\sum_{n=n(\sigma)+1}^{\infty} \exp \left\{\lambda_{n} \exp \left(-\frac{\beta\left(\lambda_{n}\right)}{\theta+\varepsilon}\right)-\lambda_{n} \sigma\right\}
\end{gathered}
$$

Where $P\left(n_{0}\right)$, the sum of first $n_{0}$ terms, is bounded. Using (10), (11) and definition of $n(\sigma)$, the above inequality gives $M(\sigma, F)$

$\leq P\left(n_{0}\right)+n(\sigma) \exp \left\{\sigma \beta^{-1}\left(-(\theta+\varepsilon) \log \frac{\sigma}{2}\right)+o(1)\right.$ 


$$
\begin{aligned}
& \leq P\left(n_{0}\right)+\bar{D} \beta^{-1}\left(-(\theta+\varepsilon) \log \frac{\sigma}{2}\right) \\
& \exp \left\{\sigma \beta^{-1}\left(-(\theta+\varepsilon) \log \frac{\sigma}{2}\right)\right\}+o(1)
\end{aligned}
$$

Or

$\log ^{+} M_{u}(\sigma, F) \leq \sigma \beta^{-1}\left(-(\theta+\varepsilon) \log \frac{\sigma}{2}\right)(1+o(1))$.

Since $\beta \in \Delta_{0}$, this easily gives

$$
\rho(\beta, F) \leq \theta
$$

The theorem now follows from (8) and (12).

\section{REFERENCES}

[1] B.L. Srivastava, A study of spaces of certain classes of Vector Valued Dirichlet. Ph. D. Thesis, I.I.T Kanpur, 1983.

[2] G.S. Srivastava and Archna Sharma , On generalized order and generalized type of vector valued Dirichlet series of slow growth. Internationa Journal of Mathematical Archive, vol. 2, pp. 2652-2659, 2011.

[3] Kong Yingying and Gan huiling, On order and type of Dirichlet series of slow growth. Turk J Math, vol. 34, pp.1-11, 2010
[4] A. Nautiyai, On the growth of annanlytic function represented by Dirichlet series. Indian J. Pure appl. Math., vol. 12,pp.1224,1981

[5] Yu Jiarong, Dirichlet series and the random Dirichlet series. Science Press, Beijing, 1997.

[6] Yu Jiarong, Some properties of random Dirichlet series. Acta Math. Sinaca, vol.21, pp:97-118, 1978.

[7] Yu Jiarong and Sun Daochun, On the distribution of values of random Dirichlet series(I). Lectures on Comp.Anal., World Scientific, Singapore, 1988.

[8] R. Ganti and G. S.Srivastava, Generalized order and generalized type of entire functions of several complex variables. Thai Journal of Mathematics, vol.4, pp: 257-265, 2006.

[9] W. C. Lu and Y. H. Peng, The infinite order of Laplace-Stieltjes transformation converging in the right plane. Pure and Applied Mathematics(in Chinese), vol.5, pp:620-627, 2012.

[10] G.S. Srivastava and A. Sharma, On bases in the space of vector valued entire Dirichlet series of two complex variables. Bulletin of Mathematical Analysis and Applications, vol.4, pp: 83-90, 2012.

[11] G.S. Srivastava and A. Sharma, Spaces of entire functions represented by vector valued Dirichlet series. Journal of Mathematics and Applications, vol.34, pp: 97-107, 2011.

[12] G.S. Srivastava and A. Sharma, Bases in the space of vector valued analytic Dirichlet series. International Journal of Pure and Applied Mathematics. Vol. 70, pp: 993-1000, 2011. 